10. Moiseev N. N. Chislennye metody v teorii optimal'nyh sistem [Numerical methods in the theory of optimal systems]. Moscow, Nauka, 1971, 424 p. (in Russian).
11. Bordovitzyna T. V. Sovremennye chislennye metody $v$ zadachah nebesnoj mehaniki [Modern numerical methods in problems of celestial mechanics]. Moscow, Nauka, 1984, 136 p. (in Russian).

Please cite this article in press as:

Kozlov E. A., Chelnokov Yu. N., Pankratov I. A. Investigation of the Problem of Optimal Correction of Angular Elements of the Spacecraft Orbit Using Quaternion Differential Equation of Orbit Orientation. Izv. Saratov Univ. (N. S.), Ser. Math. Mech. Inform., 2016, vol. 16, iss. 3, pp. 336-344 (in Russian). DOI: 10.18500/1816-9791-201616-3-336-344.

\title{
УДК 539.375
}

\section{ЩЕЛЬ ПЕРЕМЕННОЙ ШИРИНЫ ВО ВТУЛКЕ ФРИКЦИОННОЙ ПАРЫ}

\author{
В. М. Мирсалимов ${ }^{1}$, П. Э. Ахундова ${ }^{2}$ \\ ${ }^{1}$ Мирсалимов Вагифр Мирахмедович, доктор фиизико-математических наук, профрессор, заведующий кафредрой, Азербай- \\ джанский технический университет, главный научный сотрудник, Институт математики и механики НАН Азербайджана, \\ Баку, vagif.mirsalimov@imm.az \\ ${ }^{2}$ Ахундова Парвана Эльман гызы, кандидат фризико-математических наук, докторант, Институт математики и механики \\ НАН Азербайджана, Баку, sopromat_v@mail.ru \\ Рассмотрена плоская задача механики разрушения для втулки фррикционной пары. Считается, что во втулке вблизи \\ шероховатой поверхности трения имеется прямолинейная щель переменной ширины, сравнимой с упругими десормациями. \\ Дан критерий и метод решения обратной задачи механики контактного разрушения по определению фрукции перемещений \\ точек внешнего контура втулки фррикционной пары с учетом перепада температуры и неровностей контактной поверхности \\ в деталях фррикционной пары. Найденная фрункция перемещений точек внешнего контура втулки обеспечивает повышение \\ несущей способности втулки фррикционной пары.
}

Ключевые слова: фррикционная пара, втулка, плунжер, температура, шероховатая поверхность трения, щель, смыкание берегов щели, фрункция перемещений наружного контура втулки.

DOI: 10.18500/1816-9791-2016-16-3-344-355

\section{ВВЕДЕНИЕ}

Ресурс работы фрикционной пары определяется $[1,2]$ работоспособностью втулки, распределением напряжений в зонах взаимодействия деталей фрикционной пары. Практика эксплуатации фрикционных пар нефтепромыслового оборудования, транспортных машин показывает, что при многократном возвратно-поступательном движении плунжера разрушение втулки фрикционной пары происходит на пятнах фактического касания в тонких приповерхностных слоях путем образования микротрещин, с которыми втулка «живет» значительную часть ресурса работы. В связи с этим на стадии проектирования новых конструкций подвижных сопряжений нужно проводить предельный анализ деталей фрикционной пары, чтобы установить, что предполагаемые исходные щели, расположенные самым неблагоприятным образом, не будут расти до критических размеров и не вызовут разрушения в течение расчетного срока службы. Размер исходной минимальной щели следует рассматривать как проектную характеристику материала.

Считается, что внутренний контур втулки и внешний контур плунжера близки к круговым. На современном этапе развития техники важное значение имеет [3-11] оптимальное проектирование деталей фрикционной пары, обеспечивающее увеличение работоспособности фрикционных пар. Представляет значительный интерес решение задачи механики по определению такой функции перемещений точек внешнего контура втулки, при которой созданное ею напряженное поле тормозило бы развитие щели во втулке.

\section{1. ПОСТАНОВКА ЗАДАЧИ}

Рассмотрим напряженно-деформированное состояние втулки фрикционной пары. В процессе работы фрикционной пары «втулка-плунжера» при многократном возвратно-поступательном движе- 
нии плунжера происходит силовое взаимодействие между контактирующими поверхностями втулки и плунжера, возникают силы трения, приводящие к изнашиванию материалов сопряжения. Для определения контактного давления необходимо рассмотреть $[1,2]$ износоконтактную задачу о вдавливании плунжера в поверхность втулки, ослабленной прямолинейной щелью.

Пусть к внутренней поверхности втулки с упругими параметрами $G$ (модуль сдвига) и $\mu$ (коэффициент Пуассона) на некотором неизвестном заранее участке прижимается плунжер с упругими параметрами $G_{1}$ и $\mu_{1}$. Считается, что втулка на наружном контуре имеет некоторые перемещения. Функция этих перемещений заранее неизвестна и подлежит определению из дополнительного условия.

Принято, что выполняются условия плоской деформации. Режимы работа фрикционной пары, в которой могут возникать остаточные деформации, приняты недопустимыми. Пусть в упругой втулке имеется прямолинейная щель переменной ширины $h(x)$ и длиной $2 \ell_{1}$ (рис. 1$)$. Отнесем втулку фрикционной пары к полярной системе координат $r \theta$, выбрав начало координат в центре концентрических окружностей $L, L_{0}$ с радиусами $R$ и $R_{0}$ соответственно (рис. 1$)$.

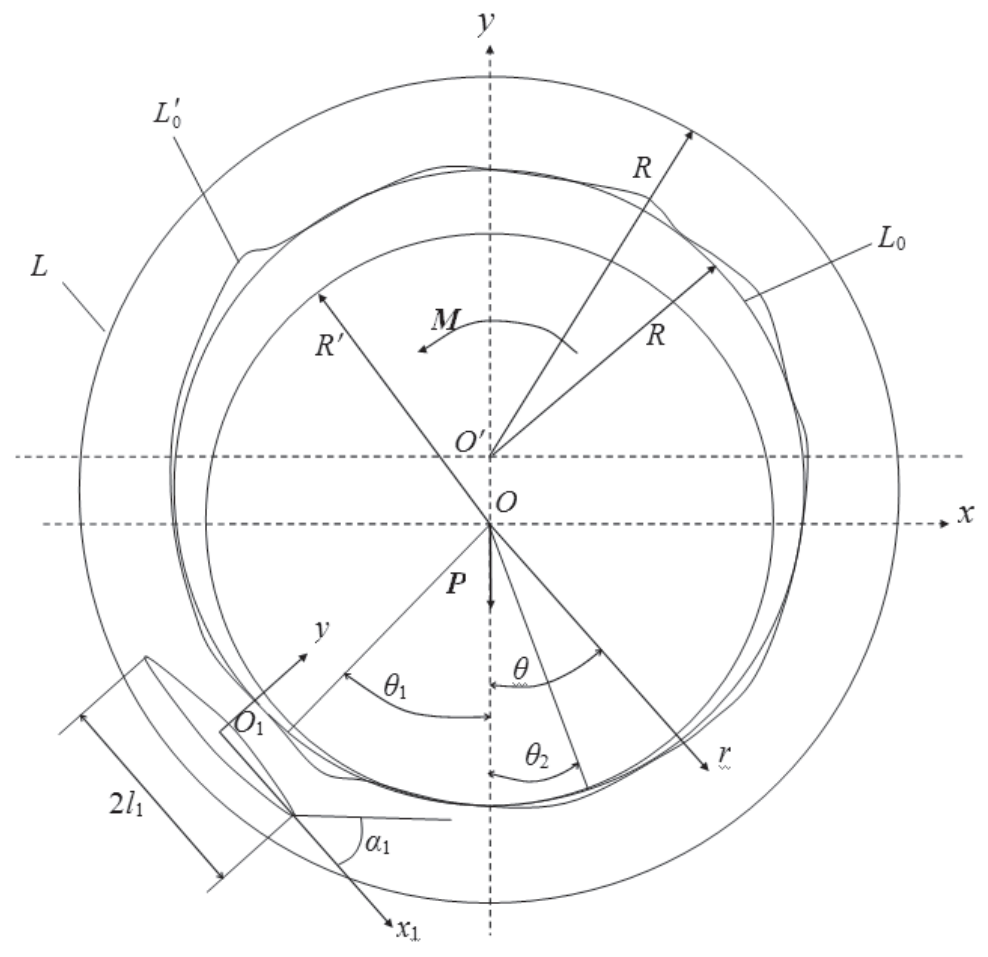

Рис. 1. Расчетная схема задачи механики разрушения для втулки фрикционной пары

Будем считать, что внутренний контур втулки и наружный контур плунжера близки к круговому. Представим границу внутреннего контура втулки $L_{0}^{\prime \prime}$ в виде $r=\rho(\theta): \rho=R_{0}+\varepsilon H(\theta)$, где $\varepsilon=R_{\max } / R_{0}-$ малый параметр; $R_{\max }-$ наибольшая высота неровности поверхности трения. С помощью профилограмм обработанной поверхности втулки находятся коэффициенты ряда Фурье для функции $H(\theta)$, описывающие каждый внутренний профиль втулки:

$$
H(\theta)=\sum_{k=0}^{n}\left(a_{k}^{0} \cos k \theta+b_{k}^{0} \sin k \theta\right) .
$$

Внешний контур плунжера близок к круговому и может быть представлен в виде

$$
\rho_{1}(\theta)=R^{\prime}+\varepsilon H_{1}(\theta), \quad H_{1}(\theta)=\sum_{k=0}^{n}\left(a_{k}^{1} \cos k \theta+b_{k}^{1} \sin k \theta\right) .
$$

Считается, что износ втулки и плунжера носит абразивный характер.

В центре прямолинейной щели разметим начало локальной системы координат $x_{1} O_{1} y_{1}$, ось $x_{1}$ которой совпадает с линией щели и образует угол $\alpha_{1}$ с осью $x$ (см. рис. 1). Принято, что берега 
щели свободны от внешних нагрузок. Условие, связывающее перемещения втулки и плунжера, имеет вид $[1,2]$ :

$$
v_{1}+v_{2}=\delta(\theta) \quad\left(\theta_{1} \leqslant \theta \leqslant \theta_{2}\right) .
$$

Здесь $\delta(\theta)$ - осадка точек поверхности втулки и плунжера, определяемая формой внутренней поверхности втулки и плунжера, а также величиной прижимающей силы $P$; $\left(\theta_{2}-\theta_{1}\right)-$ величина угла (площадки) контакта.

В зоне контакта, кроме контактных давлений, действует касательное напряжение $\tau_{r \theta}$, связанное с контактным давлением $p(\theta, t)$ по закону Амонтона - Кулона:

$$
\tau_{r \theta}=f p(\theta, t)
$$

где $f-$ коэффициент трения пары «втулка - плунжер».

Касательные усилия (силы трения) $\tau_{r \theta}(\theta, t)$ способствуют тепловыделению в зоне контакта. Общее количество тепла в единицу времени пропорционально мощности сил трения, а количество тепла, выделяемое в точке зоны контакта с координатой $\theta$, будет равно

$$
Q(\theta, t)=V f p(\theta, t)
$$

где $V-$ средняя за период скорость перемещений плунжера.

Общее количество тепла $Q(\theta, t)$ будет расходоваться следующим образом: поток тепла во втулку $Q_{b}(\theta, t)$ и аналогичный поток $Q_{1}(\theta, t)$ тепла на повышение температуры плунжера, т. е. $Q=Q_{b}+Q_{1}$.

Для перемещений точек поверхности трения втулки имеем $v_{1}=v_{1 e}+v_{1 r}+v_{1 w}$, где $v_{1 e}-$ термоупругие перемещения точек контактной поверхности втулки; $v_{1 r}, v_{1 w}$ - перемещения, вызванные смятием микровыступов и износом поверхности втулки соответственно. Аналогично для перемещений контактной поверхности плунжера имеем $v_{2}=v_{2 e}+v_{2 r}+v_{2 w}$.

Скорость изменения перемещений поверхности при износе втулки и плунжера будет равна $[1,2]$

$$
\frac{d v_{k w}}{d t}=K^{(k)} p(\theta, t) \quad(k=1,2)
$$

где $K^{(k)}-$ коэффициенты изнашивания материала втулки и плунжера $(k=1,2)$ соответственно.

Так как частота движения плунжера достаточно велика, рассматриваем задачу как стационарную. В этом случае температура втулки $(r, \theta)$ удовлетворяет дифференциальному уравнению теории темлопроводности $\Delta T=0$ и граничным условиям

$$
\begin{gathered}
\text { при } \quad r=R \quad A_{T 1} \lambda \frac{\partial T}{\partial n}-A_{T 2} \alpha_{1}^{*}\left(T-T_{c}\right)=-Q_{*}(\theta), \\
\text { при } \quad r=R_{0} \quad \lambda \frac{\partial T}{\partial n}+\alpha_{2}\left(T-T_{c}\right)=0 .
\end{gathered}
$$

Здесь $\lambda$ - коэффициент теплопроводности втулки, $\Delta-$ оператор Лапласа, $\alpha_{1}^{*}-$ коэффициент теплоотдачи с внутренней поверхности втулки, $\alpha_{2}-$ коэффициент теплоотдачи с наружной цилиндрической поверхности втулки с внешней средой с температурой $T_{c}, A_{T 1}$ - теплопоглощающая поверхность, $A_{T 2}$ - охлаждающая поверхность, $Q_{*}-$ часть количества тепла, выделившегося при трении, и приходящаяся на нагрев втулки, причем на площадке контакта $Q_{*}=Q_{b}$, а вне площадки $Q_{*}=0, n, t-$ натуральные координаты.

Для определения перемещений $v_{1}$ и $v_{1 r}$ необходимо решить задачу термоупругости для втулки при следующих условиях:

при $r=\rho(\theta)$ на площадке контакта $\sigma_{n}=-p(\theta), \tau_{n t}=-f p(\theta)$, вне площадки контакта $\sigma_{n}=0$, $\tau_{n t}=0$;

при $r=R_{0} \quad v_{r}-i v_{\theta}=g(\theta)$, на берегах щели $\sigma_{y_{1}}=0, \tau_{x_{1} y_{1}}=0$.

Здесь $\sigma_{n}, \tau_{n t}, \sigma_{y_{1}}, \tau_{x_{1} y_{1}}-$ компоненты тензора напряжений, $v_{r}, v_{\theta}-$ соответственно радиальная и касательная составляющие вектора перемещений контура $L, g(\theta)$ - искомая функция перемещений точек наружного контура $L$ втулки, $i^{2}=-1$.

Аналогично ставится задача термоупругости для определения перемещений $v_{2}$ и $v_{2 r}$ контактной поверхности плунжера

$$
\Delta T_{1}=0,
$$


при $r=\rho_{1}(\theta)$ на площадке контакта $\lambda_{1} \frac{\partial T_{1}}{\partial n}=-Q_{1}(\theta)$, вне площадки контакта $\lambda_{1} \frac{\partial T_{1}}{\partial n}+\alpha_{1}^{*} T_{1}=0$;

при $r=\rho_{1}(\theta)$ на площадке контакта $\sigma_{n}=-p(\theta), \tau_{n t}=-f p(\theta)$, вне площадки контакта $\sigma_{n}=0$, $\tau_{n t}=0$.

Контактное давление $p(\theta)$ заранее неизвестно и подлежит определению в процессе решения задачи механики контактного разрушения. Величины $\theta_{1}$ и $\theta_{2}$, являющиеся концами участка соприкосновения плунжера с втулкой, неизвестны. Для их определения используем условие [12], где давление $p(\theta)$ непрерывно переходит в нуль, когда точка $\theta$ выходит за участок соприкасания

$$
p\left(\theta_{1}\right)=0, \quad\left(\theta_{2}\right)=0
$$

Для нахождения искомой функции $g(\theta)$ перемещений точек внешнего контура $L$ втулки необходимо постановку задачи дополнить условием (критерием) определения функции $g(\theta)$. В качестве такого условия определения функции перемещений точек контура $L$ (функции $g(\theta)$ ) принимаем, что в процессе работы фрикционной пары у вершин щели должны появляться концевые зоны, берега которых смыкаются, т. е. входят в контакт. Смыкание берегов щели в концевых зонах, примыкающих к вершинам щели, сдерживает развитие щели и тем самым задерживает процесс разрушения втулки фрикционной пары.

Таким образом, требуется определить функцию перемещений точек внешнего контура $L$ втулки (функцию $g(\theta)$ ) так, чтобы созданное в процессе работы фрикционной пары напряженнодеформированное поле обеспечивало бы смыкание берегов щели в концевых зонах, примыкающих к вершинам щели. В концевых зонах, где происходит смыкание берегов щели, раскрытие щели должно обращаться в нуль

$$
\left(u_{1}^{+}-u_{1}^{-}\right)-i\left(v_{1}^{+}-v_{1}^{-}\right)=-h(x) .
$$

Это дополнительное условие позволяет определить искомую функцию $g(\theta)$ перемещений точек внешнего контура $L$ втулки.

\section{2. МЕТОД РЕШЕНИЯ}

Температуру, напряжения и перемещения во втулке и плунжере ищем в виде разложений по малому параметру, в которых пренебрегаем для упрощения членами, содержащими $\varepsilon$ степени выше первой. Каждое из приближений удовлетворяет системе дифференциальных уравнений плоской термоупругости. Значения температуры, компонент тензора напряжений при $r=\rho(\theta)$ (аналогично и при $\left.r=\rho_{1}(\theta)\right)$ получим, разлагая в ряд выражения для температуры, напряжений и перемещений в окрестности $r=R_{0}$. Используя метод возмущений с учетом сказанного, приходим к последовательности граничных условий для задач плоской теории термоупругости для втулки:

для нулевого приближения

$$
\begin{gathered}
A_{T 1} \lambda \frac{\partial t^{(0)}}{\partial r}-A_{T 2} \alpha_{1}^{*} t^{(0)}=-Q_{*}^{(0)}(\theta) \quad \text { при } \quad r=R_{0} \\
\lambda \frac{\partial t^{(0)}}{\partial r}+\alpha_{2} t^{(0)}=0 \quad \text { при } \quad r=R, \\
\sigma_{r}^{(0)}=-p^{(0)}(\theta), \quad \tau_{r \theta}^{(0)}=-f p^{(0)}(\theta) \quad \text { на площадке контакта при } r=R_{0}, \\
\sigma_{r}^{(0)}=0, \quad \tau_{r \theta}^{(0)}=0 \quad \text { вне площадки контакта, } \\
v_{r}^{(0)}-i v_{\theta}^{(0)}=g^{(0)}(\theta) \quad \text { при } \quad r=R, \\
\sigma_{y_{1}}^{(0)}=0, \quad \tau_{x_{1} y_{1}}^{(0)}=0 \quad \text { на берегах щели; }
\end{gathered}
$$

для первого приближения

$$
\begin{gathered}
A_{T 1} \lambda \frac{\partial t^{(1)}}{\partial r}-A_{T 2} \alpha_{1}^{*} t^{(1)}=-Q_{*}^{(1)}(\theta) \quad \text { при } r=R_{0}, \\
\lambda \frac{\partial t^{(1)}}{\partial r}+\alpha_{2} t^{(1)}=0 \quad \text { при } \quad r=R, \\
\sigma_{r}^{(1)}=N-p^{(1)}(\theta), \quad \tau_{r \theta}^{(1)}=T_{t}-f p^{(1)}(\theta) \quad \text { на площадке контакта при } r=R_{0}, \\
\sigma_{r}^{(0)}=N, \quad \tau_{r \theta}^{(0)}=T_{t} \quad \text { вне площадки контакта, }
\end{gathered}
$$




$$
\begin{gathered}
v_{r}^{(1)}-i v_{\theta}^{(1)}=g^{(1)}(\theta) \quad \text { при } \quad r=R, \\
\sigma_{y_{1}}^{(1)}=0, \quad \tau_{x_{1} y_{1}}^{(1)}=0 \quad \text { на берегах щели. }
\end{gathered}
$$

Здесь

$$
\begin{gathered}
Q_{*}^{(1)}(\theta)=-Q_{b}^{(1)}(\theta)+\left[A_{T 1} \lambda \frac{\partial^{2} t^{(0)}}{\partial r^{2}}-A_{T 2} \alpha_{1}^{*} \frac{\partial t^{(0)}}{\partial r}\right] H(\theta), \\
N=-H(\theta) \frac{\partial \sigma_{r}^{(0)}}{\partial r}+2 \tau_{r \theta}^{(0)} \frac{1}{R_{0}} \frac{d H(\theta)}{d \theta} \quad \text { при } r=R_{0}, \\
T_{t}=\left(\sigma_{\theta}^{(0)}-\sigma_{r}^{(0)}\right) \frac{1}{R_{0}} \frac{d H(\theta)}{d \theta}-H(\theta) \frac{\partial \tau_{r \theta}^{(0)}}{\partial r}
\end{gathered}
$$

$t=T-T_{c}-$ избыточная температура для втулки.

Аналогично можно записать граничные условия в каждом приближении для плунжера.

Дополнительные уравнения (4) принимают вид в нулевом приближении

$$
\left(u_{1}^{0+}\left(x_{1}, 0\right)-u_{1}^{0-}\left(x_{1}, 0\right)\right)-i\left(v_{1}^{0+}\left(x_{1}, 0\right)-v_{1}^{0-}\left(x_{1}, 0\right)\right)=-h(x)
$$

в первом приближении

$$
\left(u_{1}^{1+}\left(x_{1}, 0\right)-u_{1}^{1-}\left(x_{1}, 0\right)\right)-i\left(v_{1}^{1+}\left(x_{1}, 0\right)-v_{1}^{1-}\left(x_{1}, 0\right)\right)=0 .
$$

Перейдем к построению решения задачи в нулевом приближении.

Решение краевых задач теории теплопроводности в каждом приближении ищутся методом разделения переменных. Распределение избыточной температуры $t=t^{(0)}+\varepsilon t^{(1)}$ для втулки находим в следующем виде:

$$
\begin{aligned}
& t^{(0)}=C_{10}+C_{20} \ln r+\sum_{k=1}^{\infty}\left(C_{10}^{(k)} r^{k}+C_{20}^{(k)} r^{-k}\right) \cos k \theta+\sum_{k=1}^{\infty}\left(A_{10}^{(k)} r^{k}+A_{20}^{(k)} r^{-k}\right) \sin k \theta, \\
& t^{(1)}=C_{11}+C_{21} \ln r+\sum_{k=1}^{\infty}\left(C_{11}^{(k)} r^{k}+C_{21}^{(k)} r^{-k}\right) \cos k \theta+\sum_{k=1}^{\infty}\left(A_{11}^{(k)} r^{k}+A_{21}^{(k)} r^{-k}\right) \sin k \theta .
\end{aligned}
$$

Постоянные $C_{10}, C_{20}, C_{10}^{(k)}, C_{20}^{(k)}, A_{10}^{(k)}, A_{20}^{(k)}$ определяются из граничных условий (5) задачи теории теплопроводности в нулевом приближении. Соответственно коэффициенты $C_{11}, C_{21}, C_{11}^{(k)}, C_{21}^{(k)}, A_{11}^{(k)}$, $A_{21}^{(k)}$ находятся из краевых условий (7) задачи теории теплопроводности в первом приближении. Из-за громоздкости соответствующие формулы не приводятся.

Для решения задачи термоупругости в каждом приближении используем термоупругий потенциал перемещений [13]. В рассматриваемой задаче термоупругий потенциал перемещений $F$ для втулки в нулевом и первом приближениях определяется решением дифференциальных уравнений:

$$
\Delta F^{(0)}=\beta t^{(0)}, \quad \Delta F^{(1)}=\beta t^{(1)}, \quad \beta=\frac{1+\mu}{1-\mu} \alpha .
$$

Здесь $\alpha-$ коэффициент линейного температурного расширения.

Ищем решения уравнения (10) в виде

$$
\begin{gathered}
F^{(0)}=\sum_{n=0}^{\infty}\left(f_{n}^{0}(r) \cos n \theta+f_{n}^{0 *}(r) \sin n \theta\right), \\
F^{(1)}=\sum_{n=0}^{\infty}\left(f_{n}^{(1)}(r) \cos n \theta+f_{n}^{(1) *}(r) \sin n \theta\right) .
\end{gathered}
$$

Для функций $f_{n}^{0}(r), f_{n}^{0 *}(r)$ получаем обыкновенные дифференциальные уравнения, частные решения которых ищем методом вариации постоянных.

После определения термоупругого потенциала перемещений в нулевом приближении для втулки с помощью известных формул [13] вычисляем соответствующие термоупругому потенциалу напряжения $\bar{\sigma}_{r}^{(0)}, \bar{\sigma}_{\theta}^{(0)}, \bar{\tau}_{r \theta}^{(0)}$ и перемещения $\bar{v}_{r}^{(0)}, \bar{v}_{\theta}^{(0)}$ во втулке. 
Найденные напряжения и перемещения для втулки не будут удовлетворять краевым условиям (6). Необходимо для втулки найти второе напряженно-деформированное состояние $\overline{\bar{\sigma}}_{r}^{(0)}, \overline{\bar{\sigma}}_{\theta}^{(0)}, \overline{\bar{\tau}}_{r \theta}^{(0)}$ и $\overline{\bar{v}}_{r}^{(0)}$, $\overline{\bar{v}}_{\theta}^{(0)}$ такое, чтобы выполнялись граничные условия (6).

Следовательно, для определения второго напряженно-деформированного состояния имеем граничные условия:

$$
\overline{\bar{\sigma}}_{r}^{(0)}=-p^{(0)}(\theta)-\bar{\sigma}_{r}^{(0)}, \quad \overline{\bar{\tau}}_{r \theta}^{(0)}=-f p^{(0)}(\theta)-\bar{\tau}_{r \theta}^{(0)}
$$

на площадке контакта при $r=R_{0}$

$$
\begin{gathered}
\overline{\bar{\sigma}}_{r}^{(0)}=-\bar{\sigma}_{r}^{(0)}, \quad \overline{\bar{\tau}}_{r \theta}^{(0)}=-\bar{\tau}_{r \theta}^{(0)} \quad \text { вне площадки контакта, } \\
\overline{\bar{v}}_{r}^{(0)}-i \overline{\bar{v}}_{\theta}^{(0)}=g^{(0)}(\theta)-\left(\bar{v}_{r}^{(0)}-i \bar{v}_{\theta}^{(0)}\right) \quad \text { при } \quad r=R, \\
\overline{\bar{\sigma}}_{y_{1}}^{(0)}=-\bar{\sigma}_{y_{1}}^{(0)}, \quad \overline{\bar{\tau}}_{x_{1} y_{1}}^{(0)}=-\bar{\tau}_{x_{1} y_{1}}^{(0)} \quad \text { на берегах щели. }
\end{gathered}
$$

Краевые условия задачи (11)-(12) с помощью формул Колосова - Мусхелишвили [12] можно записать в виде граничной задачи для отыскания комплексных потенциалов $\Phi^{(0)}(z), \Psi^{(0)}(z)$ для втулки. Комплексные потенциалы ищем в виде

$$
\begin{gathered}
\Phi^{(0)}(z)=\Phi_{1}^{(0)}(z)+\Phi_{2}^{(0)}(z), \quad \Psi^{(0)}(z)=\Psi_{1}^{(0)}(z)+\Psi_{2}^{(0)}(z) \\
\Phi_{1}^{(0)}(z)=\sum_{k=-\infty}^{\infty} a_{k} z^{k}, \quad \Psi_{1}^{(0)}(z)=\sum_{k=-\infty}^{\infty} b_{k} z^{k} \\
\Phi_{2}^{(0)}(z)=\frac{1}{2 \pi} \int_{-\ell_{1}}^{\ell_{1}} \frac{g_{1}^{0}(t) d t}{t-z_{1}} \\
\Psi_{2}^{(0)}(z)=\frac{1}{2 \pi} e^{-2 i \alpha_{1}} \int_{-\ell_{1}}^{\ell_{1}}\left[\frac{\overline{g_{1}^{0}(t)}}{t-z_{1}}-\frac{\bar{T}_{1} e^{i \alpha_{1}}}{\left(t-z_{1}\right)^{2}} g_{1}^{0}(t)\right] d t .
\end{gathered}
$$

Здесь $T_{1}=t e^{i \alpha_{1}}+z_{1}^{0}, z_{1}=e^{-i \alpha_{1}}\left(z-z_{1}^{0}\right), g_{1}^{0}(t)$ - искомая функция, характеризующая скачок перемещений в нулевом приближении при переходе через линию щели.

$$
\begin{gathered}
g_{1}(x)=\frac{2 G}{i(1+\kappa)} \frac{\partial}{\partial x_{1}}\left[u_{1}^{0+}\left(x_{1}, 0\right)-u_{1}^{0-}\left(x_{1}, 0\right)+i\left(v_{1}^{0+}\left(x_{1}, 0\right)-v_{1}^{0-}\left(x_{1}, 0\right)\right)\right], \\
\kappa=3-4 \mu .
\end{gathered}
$$

Представим граничную задачу для отыскания комплексных потенциалов на круговых границах в следующем виде:

$$
\begin{gathered}
\Phi_{1}^{(0)}\left(\tau_{0}\right)+\overline{\Phi_{1}^{(0)}\left(\tau_{0}\right)}-e^{2 i \theta}\left[\bar{\tau}_{0} \Phi_{1}^{(0)}\left(\tau_{0}\right)+\Psi_{1}^{(0)}\left(\tau_{0}\right)\right]=X^{(0)}(\theta)-\left(f_{1}-i f_{2}\right), \\
\Phi_{1}^{(0)}(\tau)-\kappa \overline{\Phi_{1}^{(0)}(\tau)}-e^{2 i \theta}\left[\bar{\tau} \Phi_{1}^{\prime(0)}(\tau)+\Psi_{1}^{(0)}(\tau)\right]=g^{(0)}(\theta)-\left(f_{3}-i f_{4}\right), \\
\tau=R \exp (i \theta) \quad \tau_{0}=R_{0} \exp (i \theta), \\
X^{(0)}(\theta)= \begin{cases}-(1-i f) p^{(0)}(\theta)-\left(\bar{\sigma}_{r}^{(0)}-i \bar{\tau}_{r \theta}^{(0)}\right) & \text { на площадке контакта, } \\
-\left(\bar{\sigma}_{r}^{(0)}-i \bar{\tau}_{r \theta}^{(0)}\right) & \text { вне площадки контакта. }\end{cases}
\end{gathered}
$$

Для решения краевой задачи (14) относительно потенциалов $\Phi_{1}^{(0)}(z)$ и $\Psi_{1}^{(0)}(z)$ используется метод степенных рядов. Для этого правые части условий (14) разлагаются в ряды Фурье

$$
\begin{gathered}
X^{(0)}(\theta)=\sum_{k=-\infty}^{\infty} A_{k}^{(0)} e^{i k \theta} \\
-\left(f_{1}-i f_{2}\right)=\sum_{k=-\infty}^{\infty} D_{k}^{(0)} e^{i k \theta}, \quad-\left(f_{3}-i f_{4}\right)=\sum_{k=-\infty}^{\infty} F_{k}^{(0)} e^{i k \theta} .
\end{gathered}
$$

Коэффициенты $D_{k}^{(0)}$ и $F_{k}^{(0)}$ выражаются в виде интегралов от искомой функции $g_{1}^{(0)}$. Для их определения использовали теорию вычетов. После некоторых преобразований придем к бесконечной 
линейной алгебраической системе относительно коэффициентов $a_{k}$ и $b_{k}$. В эту систему входят коэффициенты разложения функции $g^{(0)}(\theta)$ перемещений точек внешнего контура $L$ втулки в нулевом приближении

$$
g^{(0)^{\prime}}(\tau)=\sum_{k=-\infty}^{\infty} A_{k}^{0} e^{i k \theta}=a_{0}^{*(0)}+\sum_{k=1}^{\infty}\left(a_{k}^{*(0)} \cos k \theta+b_{k}^{*(0)} \sin k \theta\right),
$$

контактного давления

$$
p^{(0)}(\theta)=\alpha_{0}^{0}+\sum_{k=1}^{\infty}\left(\alpha_{k}^{0} \cos k \theta+\beta_{k}^{0} \sin k \theta\right),
$$

а также интегралы от искомой функции $g_{1}^{(0)}(t)$.

Удовлетворяя функциями (13) граничным условиям на берегах щели (12), получим сингулярное интегральное уравнение относительно неизвестной функции $g_{1}^{(0)}\left(x_{1}\right)$ :

$$
\begin{gathered}
\int_{-\ell_{1}}^{\ell_{1}}\left[R\left(t, x_{1}\right) g_{1}^{0}(t)+S\left(t, x_{1}\right) \overline{g_{1}^{0}(t)}\right] d t=\pi f_{1}\left(x_{1}\right) \\
f_{1}\left(x_{1}\right)=-\left[\Phi_{1}^{(0)}\left(x_{1}\right)+\overline{\Phi_{1}^{(0)}\left(x_{1}\right)}+x_{1} \overline{\Phi_{1}^{(0)^{\prime}}\left(x_{1}\right)}+\overline{\Psi_{1}^{(0)}\left(x_{1}\right)}\right]-\left(\bar{\sigma}_{y_{1}}^{(0)}-\bar{\tau}_{x_{1} y_{1}}^{(0)}\right) .
\end{gathered}
$$

Переменные $x_{1}, t, \ell_{1}$ - безразмерные величины, отнесенные к $R_{0} ; R\left(t, x_{1}\right)$ и $S\left(t, x_{1}\right)$ определяются по известным соотношениям [14, формулы (VI.61)].

K сингулярному интегральному уравнению для внутренней щели следует добавить условие однозначности перемещений при обходе контура щели

$$
\int_{-\ell_{1}}^{\ell_{1}} g_{1}^{0}(t) d t=0
$$

С помощью процедуры алгебраизации [14-16] комплексное сингулярное интегральное уравнение (15) при отмеченном выше условии (16) сводится к системе $M$ комплексных алгебраических уравнений для определения $M$ неизвестных $g_{1}^{0}\left(t_{m}\right)=v_{1}^{0}\left(t_{m}\right)-i u_{1}^{0}\left(t_{m}\right)(m=1,2, \ldots, M)$

$$
\begin{gathered}
\frac{1}{M} \sum_{m=1}^{M} \ell_{1}\left[g_{1}^{0}\left(t_{m}\right) R\left(\ell_{1} t_{m}, \ell_{1} x_{r}\right)+\overline{g_{1}^{0}\left(t_{m}\right)} S\left(\ell_{1} t_{m}, \ell_{1} x_{r}\right)\right]=f_{1}\left(x_{r}\right), \\
\sum_{m=1}^{M} g_{1}^{0}\left(t_{m}\right)=0, \quad r=1,2, \ldots, M-1 \\
t_{m}=\cos \frac{2 m-1}{2 M} \pi, \quad x_{r}=\cos \frac{\pi r}{M} .
\end{gathered}
$$

Если в (17) перейти к комплексно сопряженным значениям, получим еще $M$ алгебраических уравнений. В правые части системы (17) входят неизвестные значения коэффициентов разложения функций перемещений точек внешнего контура $L$ втулки и контактного давления $p^{(0)}(\theta)$.

С помощью комплексных потенциалов (13), формул Колосова - Мусхелишвили и интегрирования кинетического уравнения изнашивания (2) материала втулки находится радиальное перемещение $v_{1}^{0}$ контактной поверхности втулки в нулевом приближении.

Аналогично рассматривается задача термоупругости для плунжера. С использованием решения задачи термоупругости для плунжера в нулевом приближении и кинетического уравнения изнашивания материала плунжера находится радиальное перемещение $v_{2}^{0}$ контактной поверхности плунжера в нулевом приближении. Найденные величины $v_{1}^{0}$ и $v_{2}^{0}$ подставляются в основное контактное уравнение (1) в нулевом приближении.

Для алгебраизации основного контактного уравнения неизвестные функции контактного давления в нулевом приближении ищутся в виде разложений

$$
\begin{gathered}
p^{(0)}(\theta, t)=p_{0}^{0}(\theta)+t p_{1}^{0}(\theta)+\ldots, \\
p_{s}^{0}(\theta)=\alpha_{0}^{s}+\sum_{k=1}^{\infty}\left(\alpha_{k}^{s} \cos k \theta+\beta_{k}^{s} \sin k \theta\right), \quad s=0,1, \ldots
\end{gathered}
$$


Подставляя соотношения (18) в основное контактное уравнение в нулевом приближении, получим функциональные уравнения для последовательного определения $p_{0}^{0}(\theta), p_{1}^{0}(\theta)$ и т.д. Для построения алгебраической системы относительно $\alpha_{k}, \beta_{k}$ приравниваем коэффициенты при одинаковых тригонометрических функциях в левой и правой частях функционального уравнения контактной задачи. В результате получим бесконечную алгебраическую систему относительно $\alpha_{k}^{0}(k=0,1,2, \ldots), \beta_{k}^{0}$ $(k=1,2, \ldots)$ и $\alpha_{k}^{1}, \beta_{k}^{1}$ и т. д. Из-за неизвестных величин $\theta_{1}$ и $\theta_{2}$ система уравнений оказывается нелинейной. Для определения величин $\theta_{1}$ и $\theta_{2}\left(\theta_{1}=\theta_{1}^{0}+\varepsilon \theta_{1}^{1}+\ldots ; \theta_{2}=\theta_{2}^{0}+\varepsilon \theta_{2}^{1}+\ldots\right)$ имеем условие (3). Эти уравнения можно представить в виде

$$
\begin{array}{ll}
p^{(0)}\left(\theta_{1}^{0}\right)=0, & p^{(0)}\left(\theta_{2}^{0}\right)=0 \quad \text { для нулевого приближения, } \\
p^{(1)}\left(\theta_{1}^{1}\right)=0, & p^{(1)}\left(\theta_{2}^{1}\right)=0 \quad \text { для первого приближения. }
\end{array}
$$

В правые части бесконечных алгебраических систем относительно $\alpha_{k}, \beta_{k}$ входят интегралы от неизвестной функции $g_{1}^{0}\left(x_{k}\right)$, а также неизвестные значения коэффициентов разложения функции $g^{0}(\theta)$ перемещений точек внешнего контура $L$ втулки в нулевом приближении. Таким образом, бесконечные алгебраические системы относительно $\alpha_{k}, \beta_{k}$ и конечная система относительно $g_{1}^{0}\left(x_{k}\right)$ связаны между собой и их надо решать совместно.

Полученные системы уравнений относительно $a_{k}, b_{k}, \alpha_{k}, \beta_{k}, g_{1}^{0}\left(t_{m}\right)(m=1,2, \ldots, M)$ позволяют при заданной функции перемещений точек внешнего контура $L$ втулки найти в нулевом приближении напряженно-деформированное состояние втулки фрикционной пары при наличии щели во втулке, контактное давление, коэффициенты интенсивности напряжений в окрестности вершин щели, распределение температуры, а также абразивный износ деталей фрикционной пары. В поставленной задаче оптимального проектирования требуется определить функцию перемещений точек внешнего контура $L$ втулки. Коэффициенты $A_{k}^{0}(k=0, \pm 1, \pm 2, \ldots)$ подлежат определению. Следовательно, полученная объединенная алгебраическая система не является пока замкнутой.

Для построения недостающих уравнений в нулевом приближении требуем выполнения условий (8) в узловых точках, принадлежащих концевым зонам, в которых должно происходить смыкание берегов щели. В рассматриваемом случае вместо (8) удобно использовать выражение для производной раскрытия берегов щели. Таким образом, недостающие уравнения в нулевом приближении получаются в виде

$$
g_{1}^{0}\left(t_{k}\right)=-\frac{2 G}{1+\kappa} h^{\prime}\left(t_{k}\right), \quad k=1,2, \ldots, M_{1},
$$

где $M_{1}-$ число узловых точек, принадлежащих концевым зонам.

\section{3. МЕТОДИКА ЧИСЛЕННОГО РЕШЕНИЯ}

Совместное решение полученных систем уравнений позволяет найти приближенные значения коэффициентов $a_{k}, b_{k}, \alpha_{k}, \beta_{k}$, значений функций раскрытия берегов щели $v_{1}^{0}\left(t_{m}\right), u_{1}^{0}\left(t_{m}\right)$ и коэффициентов функции перемещений точек внешнего контура $L$ втулки $A_{k}^{0}\left(k=0, \pm 1, \pm 2, \ldots, \pm M_{1}\right)$. Так как размеры концевых зон мы задаем заранее, то система (21) уравнений оказывается линейной. Объединенная же система алгебраических уравнений из-за неизвестных величин $\theta_{1}^{0}$ и $\theta_{2}^{0}$ будет нелинейной. Для ее решения используется методы редукции и последовательных приближений, суть которых состоит в следующем. Решаем объединенную алгебраическую систему при некоторых определенных значениях $\theta_{1}^{0 *}$ и $\theta_{2}^{0 *}$ относительно остальных неизвестных (были перечислены выше). Остальные неизвестные входят в объединенную систему линейным образом. Значения $\theta_{1}^{0 *}$ и $\theta_{2}^{0 *}$ и найденные величины остальных неизвестных подставляются в неиспользованные уравнения (19). Взятые значения $\theta_{1}^{0 *}$ и $\theta_{2}^{0 *}$ и соответствующие им значения остальных неизвестных не будут, вообще говоря, удовлетворять уравнениям (19). Поэтому, подбирая значения параметров $\theta_{1}^{0}$ и $\theta_{2}^{0}$, будем многократно повторять вычисления до тех пор, пока последние уравнения системы (19), (20) не будут удовлетворяться с заданной точностью.

После определения искомых величин нулевого приближения можно перейти к построению решения обратной задачи в первом приближении. На основании полученного решения при $R_{0}$ определяются функции $N$ и $T_{t}$. Краевые условия (7) записываются в виде граничной задачи для отыскания комплексных потенциалов $\Phi^{(1)}(z)$ и $\Psi^{(1)}(z)$. Комплексные потенциалы $\Phi^{(1)}(z)$ и $\Psi^{(1)}(z)$ ищем аналогично как (13) с очевидными изменениями. Дальнейший ход решения такой же, как и в нулевом приближении. Полученное комплексное сингулярное интегральное уравнение относительно $g_{1}^{1}(t)$ при 
дополнительном условии типа (16) с помощью процедуры алгебраизации сводится к конечной системе $M$ алгебраических уравнений для определения $M$ неизвестных $g_{1}^{1}\left(t_{m}\right) \quad(m=1,2, \ldots, M)$.

В правые части этой системы входят коэффициенты разложения функции $g^{(1)}(\theta)$ перемещений точек внешнего контура $L$ втулки в первом приближении

$$
g^{(1)^{\prime}}(\theta)=\sum_{k=-\infty}^{\infty} A_{k}^{1} e^{i k \theta}=a_{0}^{*(1)}+\sum_{k=1}^{\infty}\left(a_{k}^{*(1)} \cos k \theta+b_{k}^{*(1)} \sin k \theta\right)
$$

контактного давления $p^{(1)}(\theta)=\alpha_{0}^{1}+\sum_{k=1}^{\infty}\left(\alpha_{k}^{1} \cos k \theta+\beta_{k}^{1} \sin k \theta\right)$, а также интегралы от функции $g_{1}^{1}(t)$.

Построение недостающих уравнений для определения неизвестных контактных напряжений осуществляется подобно нулевому приближению. Аналогично решается задача термоупругости для плунжера в первом приближении. Алгебраизация основного уравнения контактной задачи в первом приближении проводится таким же образом, как и в нулевом приближении. Для этого искомые функции контактного давления представляются в виде

$$
\begin{gathered}
p^{(1)}(\theta)=p_{0}^{1}(\theta)+t p_{0}^{1}(\theta)+\ldots, \\
p_{0}^{1}(\theta)=\alpha_{0,0}^{1}+\sum_{k=1}^{\infty}\left(\alpha_{k, 0}^{1} \cos k \theta+\beta_{k, 0}^{1} \sin k \theta\right), \quad p_{1}^{1}(\theta)=\alpha_{0,1}^{1}+\sum_{k=1}^{\infty}\left(\alpha_{k, 1}^{1} \cos k \theta+\beta_{k, 1}^{1} \sin k \theta\right) .
\end{gathered}
$$

В результате получим бесконечные линейные алгебраические системы относительно $\alpha_{0,0}^{1}, \alpha_{k, 0}^{1}$, $\beta_{k, 0}^{1}(k=1,2, \ldots)$ и $\alpha_{0,1}^{1}, \alpha_{k, 1}^{1}, \beta_{k, 1}^{1}(k=1,2, \ldots, n)$.

Из-за неизвестных величин $\theta_{1}^{1}$ и $\theta_{2}^{1}$ система уравнений оказывается нелинейной.

Полученные системы уравнений относительно $a_{k}^{1}, b_{k}^{1}, \alpha_{k}^{1}, \beta_{k}^{1}, g_{1}^{1}\left(t_{m}\right)(m=1,2, \ldots, M)$ позволяют при заданной функции перемещений точек внешнего контура $L$ втулки найти в первом приближении напряженно-деформированное состояние втулки фрикционной пары при наличии щели во втулке, контактное давление, коэффициенты интенсивности напряжений в окрестности вершин щели, распределение температуры, а также абразивный износ втулки и плунжера.

В рассматриваемой задаче оптимального проектирования коэффициенты $A_{k}^{1}(k=0, \pm 1, \pm 2, \ldots)$ функции перемещений точек внешнего контура $L$ втулки подлежат определению. Для построения недостающих уравнений в первом приближении требуем выполнения дополнительных условия (9) в узловых точках, принадлежащих концевым зонам, в которых происходит смыкание берегов щели. Как и в нулевом приближении, вместо (9) удобно использовать соотношение для производной раскрытия берегов щели. Следовательно, недостающие уравнения в первом приближении имеем в следующем виде:

$$
g_{1}^{1}\left(t_{k}\right)=0, \quad k=1,2, \ldots, M_{1}
$$

\section{4. ЧИСЛЕННЫЕ РЕЗУЛЬТАТЫ И ИХ АНАЛИЗ}

Из-за неизвестных концов контактной площадки (величин $\theta_{1}$ и $\theta_{2}$ ) объединенная система уравнений оказывается нелинейной. Построенная объединенная система уравнений является замкнутой и позволяет при заданных функциях $H(\theta)$ и $H_{1}(\theta)$ численными расчетами найти оптимальную функцию перемещений точек внешнего контура $L$ втулки, контактное давление, напряженно-деформированное состояние, температуру и износ втулки и плунжера фрикционной пары.

Функции $H(\theta)$ и $H_{1}(\theta)$, описывающие шероховатости внутренней поверхности втулки и плунжера, рассматривались [17-20] как детермированные совокупности неровностей профиля контуров и моделировались стационарной случайной функцией с нулевым средним значением и известной дисперсией.

В рассматриваемой задаче имеется много свободных параметров. Это различные теплофизические и механические характеристики материалов, геометрические размеры втулки, скорость движения плунжера. Результаты расчета контактного давления для втулки бурового насоса в зависимости от 
значения полярного угла $\theta^{\prime}=\theta-\theta_{+}$ $\left(\theta_{0}=\frac{\theta_{2}-\theta_{1}}{2}, \theta_{+}=\frac{\theta_{2}+\theta_{1}}{2}\right)$ представлены в виде графиков на рис. 2 при скорости плунжера $V=0.4 \mathrm{~m} / \mathrm{c}$ для случая прямолинейной щели при $\alpha_{1}=60^{\circ}$; кривая 1 соответствует шероховатому контуру, а кривая 2 - гладкому. В качестве параметров были приняты: $2 R_{0}=57 \mathrm{мм}$, $2 R=73 \mathrm{Mм}, 2 R_{0}^{\prime}=56.7 \mathrm{mм}$, $f=0.2, E=1.8 \cdot 10^{5} \mathrm{M \Pi а}$, $E_{1}=2.1 \cdot 10^{5} \mathrm{M \Pi а}, \nu=0.25, \nu_{1}=0.3$, $K^{(1)}=2 \cdot 10^{-8}, K^{(2)}=2.5 \cdot 10^{-9}$, $\Delta=R_{0}-R_{0}^{\prime}=0.15$ мм, ширина щели меняется по параболическому закону.

Максимальные значения контактного давления, как правило, находятся в средней части контактной поверхности в зависимости от угла обхвата и коэффициента трения. Наличие сил трения в контактной зоне приводит к смещению графика распределения контактного давления в сторону, противоположную действию момента.

Результаты расчетов функции перемещений точек внешнего контура $L$ втулки приведены в таблице.

\begin{tabular}{|c|c|c|c|c|c|c|c|c|c|}
\hline$a_{0}^{*}$ & $a_{1}^{*}$ & $a_{2}^{*}$ & $a_{3}^{*}$ & $a_{4}^{*}$ & $a_{5}^{*}$ & $a_{6}^{*}$ & $a_{7}^{*}$ & $a_{8}^{*}$ & $a_{9}^{*}$ \\
\hline 0.1152 & 0.0897 & 0.0754 & 0.0671 & 0.0582 & 0.0497 & 0.0412 & 0.0317 & 0.0291 & 0.0213 \\
\hline & $b_{1}^{*}$ & $b_{2}^{*}$ & $b_{3}^{*}$ & $b_{4}^{*}$ & $b_{5}^{*}$ & $b_{6}^{*}$ & $b_{7}^{*}$ & $b_{8}^{*}$ & $b_{9}^{*}$ \\
\hline & 0.0825 & 0.0713 & 0.0589 & 0.0516 & 0.0432 & 0.0367 & 0.0281 & 0.0214 & 0.0147 \\
\hline
\end{tabular}

Примечание. $V=0.4$ м/с, коэффициенты даны в мм.

Меняя значения параметров $z_{1}^{0}$ и $\alpha_{1}$, характеризующих положение щели, можно исследовать различные случаи расположения щели во втулке. Если же щель одним концом выходит на внутреннюю поверхность втулки, то равенство (16) заменяется условием, выражающим конечность напряжений у края щели.

Там, где берега щели вошли в контакт, т.е. в концевых областях, возникнут нормальные $q_{y_{1}}\left(x_{1}\right)$ и касательные $q_{x_{1} y_{1}}\left(x_{1}\right)$ контактные напряжения. Для их определения при известной уже функции перемещений $g(\theta)$ точек внешнего контура $L$ втулки необходимо вновь решить задачу механики разрушения для щели с частично контактирующими берегами. Метод решения таких задач для втулки фрикционной пары дан в [21].

\section{5. выводы}

Практика эксплуатации фрикционных пар показывает, что на стадии проектирования новых конструкций подвижных сопряжений необходимо учитывать случаи, когда в отдельных узлах трения (втулка) могут возникнуть щели. Полученные в статье основные разрешающие уравнения позволяют при заданной функции перемещений точек внешнего контура втулки численными расчетами путем нахождения коэффициентов интенсивности напряжений прогнозировать рост имеющейся щели во втулке, установить допустимый размер дефекта и максимальные значения рабочих нагрузок, обеспечивающих достаточный запас надежности. Решение обратной задачи по определению функции перемещений точек внешнего контура втулки дает возможность на стадии проектирования устанавливать оптимальные геометрические параметры элементов подвижных сопряжений, обеспечивающие повышение несущей способности фрикционной пары.

\section{Библиографический список}

1. Горячева И. Г., Добычин М. Н. Контактные задачи в трибологии. М. : Машиностроение, 1988. 256 с.

2. Горячева И. Г Механика фрикционного взаимодействия. М. : Наука, 2001. 478 с.
3. Гаджиев Г. Х., Мирсалимов В. М Оптимальное проектирование контактной пары цилиндрплунжер // Трение и износ. 2004. Т. 25, № 5. C. $466-473$. 
4. Мирсалимов В. М. Обратная задача механики разрушения для составного цилиндра // Изв. РАН MTT. 2009. № 1. С. 165-173.

5. Мирсалимов В. М. Минимизация теплового состояния втулки контактной пары // Проблемы машиностроения и надежности машин. 2006. № 5. C. $88-95$

6. Мирсалимов В. М. Минимизация напряженного состояния втулки контактной пары // Трение и износ. 2006. Т. 27, № 4. С. 388-393.

7. Мирсалимов В. М. Обратная износоконтактная задача для фрикционной пары // Проблемы машин и надежности машин. 2008. № 1. С. 62-69.

8. Мирсалимов В. М. Обратная задача механики разрушения для диска, посаженного на вращающийся вал // Прикладная механика и техническая физика. 2009. Т. 50, № 4. С. 201-209.

9. Mirsalimov V. M., Veliyev F. E. Inverse problem of failure mechanics for a drawing die strengthened with a holder // Acta Polytechnica Hungarica. 2013. Vol. 10. P. 121-138. DOI: 10.12700/APH.10.01.2013.1.7.

10. Гаджиев Г. Х., Мирсалимов В. М. Минимизация износа внутренней поверхности втулки составного цилиндра контактной пары // Трение и износ. 2004. T. 25, № 3. С. 231-237.

11. Мирсалимов В. М., Ахундова П. Э. Минимизация контактного давления для фрикционной пары «втулка - вал» // Трение и износ. 2015. Т. 36, № 5. C. 529-535.

12. Мусхелишвили Н. И. Некоторые основные задачи математической теории упругости. М. : Наука, 1966. 707 c.
13. Паркус $Г$. Неустановившиеся температурные напряжения. М. : Физматгиз, 1963. 252 с.

14. Панасюк В. В., Саврук М. П., Дацьышин А. П. Распределение напряжений около трещин в пластинах и оболочках. Киев : Наук. думка, 1976. $443 \mathrm{c}$.

15. Мирсалимов В. М. Неодномерные упругопластические задачи. М. : Наука, 1987. 256 с.

16. Ladopoulos E. G. Singular integral equations : Linear and non-linear theory and its Applications in Science and Engineering. Berlin : Springer-Verlag, 2000. 556 p. DOI: 10.1007/978-3-662-04291-5.

17. Thomas T. R. Rough surface. L. : Longman, 1982. $261 \mathrm{p}$.

18. Bhushan B. Contact mechanics of rough surfaces in tribology : multiple asperity contact // Tribology Letters. 1998. Vol. 4, iss. 1. P. 1-35. DOI: 10.1023/A:1019186601445

19. Carbone G., Bottiglione F. Contact mechanics of rough surfaces : a comparison between theories // Meccanica. 2011. Vol. 46, iss. 3. P. 557-565. DOI: 10.1007/s11012-010-9315-y.

20. Czifra A., Horvath S. Complex microtopography analysis in sliding friction of steel-ferodo material pair // Meccanica. 2011. Vol. 46, iss. 3. P. 609-616. DOI: $10.1007 / \mathrm{s} 11012-010-9422-9$.

21. Мирсалимов В. М. Моделирование закрытия трещины со связями между берегами во втулке контактной пары // Изв. РАН. МТТ. 2009. № 2. C. $78-92$.

\title{
Образец для цитирования:
}

Мирсалимов В. М., Ахундова П. Э. Щель переменной ширины во втулке фрикционной пары // Изв. Сарат. ун-та. Нов. сер. Сер. Математика. Механика. Информатика. 2016. Т. 16, вып. 3. С. 344-355. DOI: 10.18500/1816-97912016-16-3-344-355.

\section{Slot of Variable Width in a Hub of Friction Pair}

\section{M. Mirsalimov ${ }^{1}$, P. E. Akhundova ${ }^{2}$}

\author{
${ }^{1}$ Vagif M. Mirsalimov, Institute of Mathematics and Mechanics of NAS of Azerbaijan, 9, B. Vahabzade, Baku, Azerbaijan, AZ1141; \\ Azerbaijan Technical University 25, H. Javid av., Baku, Azerbaijan, AZ1073, vagif.mirsalimov@imm.az \\ ${ }^{2}$ Parvana E. Akhundova, Institute of Mathematics and Mechanics of NAS of Azerbaijan, 9, B. Vahabzade, Baku, Azerbaijan, AZ1141, \\ sopromat_v@mail.ru
}

\begin{abstract}
Plane problem of fracture mechanics for a hub of a friction pair is studied. It is suggested that near the rough friction surface, the hub has a rectilinear slot of variable width. The slot width is comparable with elastic deformations. A criterion and a method for solving the inverse problem of mechanics of contact fracture on definition of displacement function of the hub external contour points in a friction pair with regard to the temperature drop and irregularities of the contact surface in friction pair components is given. The obtained displacement function of the external contour points of the hub provides the increase of load-bearing capacity of the friction pair hub.

Key words: friction pair, hub, plunger, temperature, rough friction surface, slot, closure of slot faces, function of displacements of external contour of the hub.
\end{abstract}




\section{References}

1. Goryacheva I. G. Contact mechanics in tribology. Dordrecht, Kluwer. Acad. Publ., 1998. 346 p. DOI: 10.1007/978-94-015-9048-8 (Russ. ed.: Goryacheva I. G., Dobychin M. N. Kontaktnye zadachi v tribologii. Moscow, Mashinostroenie, 1988, 256 p.)

2. Goryacheva I. G. Mehanika frikcionnogo vzaimodejstvija [Mechanics of frictional interaction]. Moscow, Nauka, 2001, 478 p. (in Russian).

3. Gadzhiev G. H., Mirsalimov V. M. Optimal design of the composite cylinder-piston contacting pair. J. of Friction and Wear, 2004, vol. 25, no. 5, pp. 466-473.

4. Mirsalimov V. M. Inverse problem of fracture mechanics for a compound cylinder. Mech. Solids, 2009, vol. 44, iss. 1, pp. 141-148. DOI : 10.3103/S0025654409010154.

5. Mirsalimov V. M. Minimization of thermal state of contact pair bushing. Problemy Mashinostroeniya i Nadezhnosti Mashin, 2006, no. 5, pp. 88-95 (in Russian).

6. Mirsalimov V. M. Minimization of stress state of contact pair bushing. J. of Friction and Wear, 2006, vol. 27, no. 4, pp. 388-393.

7. Mirsalimov V. M. An inverse wear contact problem for a friction couple. J. of Machinery Manufacture and Reliability, 2008, vol. 37, iss. 1, pp. 53-59. DOI: 10.1007/s12001-008-1011-2.

8. Mirsalimov V. M. Inverse problem of fracture mechanics for a disk fitted onto a rotating shaft. $J$. of Applied Mech. and Technical Physics, 2009, vol. 50, iss. 4, pp. $712-719$. DOI: $10.1007 / \mathrm{s} 10808$ 009-0097-8.

9. Mirsalimov V. M., Veliyev F. E. Inverse problem of failure mechanics for a drawing die strengthened with a holder. Acta Polytechnica Hungarica, 2013, vol. 10, no. 1, pp. 121-138. DOI: 10.12700/APH.10.01.2013.1.7.

10. Gadzhiev G. H., Mirsalimov V. M. Minimizing wear of the internal surface of a split cylinder sleeve in a contact pair. J. of Friction and Wear, 2004, vol. 25, no. 3, pp. 231-237.
11. Mirsalimov V. M., Akhundova P. E. Minimization of contact pressure for hub-shaft friction pair. J. of Friction and Wear, 2015, vol. 36, iss. 5, pp. 404408. DOI: 10.3103/S1068366615050116.

12. Muskhelishvili N. I. Some Basic Problem of Mathematical Theory of Elasticity. Amsterdam, Kluwer, 1977, 732 p.

13. Parkus H. Instationäre Wärmespannungen. Wien, Springer-Verlag, 1959, 168 p. DOI: 10.1007/978-37091-5710-7.

14. Panasyuk V. V., Savruk M. P., Datsyshyn A. P. Raspredelenie naprjazhenij okolo treshhin v plastinah $i$ obolochkah [The stress distribution around cracks in plates and shells]. Kiev, Naukova Dumka, 1976, 443 p. (in Russian)

15. Mirsalimov V. M. Neodnomernye uprugoplasticheskie zadachi [Non-one-dimensional elastoplastic problems]. Moscow, Nauka, 1987, 256 p. (in Russian).

16. Ladopoulos E. G. Singular integral equations: Linear and non-linear theory and its Applications in Science and Engineering. Berlin, Springer-Verlag, 2000, 556 p. DOI: 10.1007/978-3-662-04291-5.

17. Thomas T. R. Rough surface. London, Longman, 1982, $261 \mathrm{p}$.

18. Bhushan B. Contact mechanics of rough surfaces in tribology: multiple asperity contact. Tribology Letters, 1998, vol. 4, iss. 1, pp. 1-35. DOI: 10.1023/A:1019186601445.

19. Carbone G., Bottiglione F. Contact mechanics of rough surfaces : a comparison between theories. Meccanica, 2011, vol. 46, iss. 3, pp. 557-565. DOI: 10.1007/s11012-010-9315-y.

20. Czifra A., Horvath S. Complex microtopography analysis in sliding friction of steel-ferodo material pair. Meccanica, 2011, vol. 46, iss. 3, pp. 609-616. DOI: $10.1007 / \mathrm{s} 11012-010-9422-9$.

21. Mirsalimov V. M. Simulation of bridged crack closure in a contact pair bushing. Mechanics of Solids. 2009, vol. 44, iss. 2, pp. 232-243. DOI: $10.3103 /$ S0025654409020083.

\section{Please cite this article in press as:}

Mirsalimov V. M., Akhundova P. E. Slot of Variable Width in a Hub of Friction Pair. Izv. Saratov Univ. (N. S.), Ser. Math. Mech. Inform., 2016, vol. 16, iss. 3, pp. 344-355 (in Russian). DOI: 10.18500/1816-9791-2016-16-3-344-355. 